Research Paper

\title{
The Association between Obesity and Severity in Patients with Coronavirus Disease 2019: a Retrospective, Single-center Study, Wuhan
}

\author{
Jishou Zhang, MD ${ }^{1,2,3^{*}}$, Yao Xu, MD ${ }^{1,2,3^{*}}$, Bo Shen, MD PHD ${ }^{7^{*}}$, Hua He, MD PHD ${ }^{*}$, Mingxiao Liu, MD ${ }^{6^{*}}$, \\ Mengmeng Zhao, MD1,2,3, Jianfang Liu, MD1,2,3, Shuwan Xu, MD1,2,3, Wei Pan, BS1,2,3, Jing Ye, MD1,2,3, Zhen \\ Wang, MD $1,2,3$, Di Ye, MD1,2,3, Menglin Liu, MD, Dan Li, MD5 , Zhen Luo, MD, Yongqi Feng, MD1,2,3, \\ Menglong Wang, MD PHD ${ }^{1,2,3}$, and Jun Wan, MD PHD ${ }^{1,2,3}{ }^{\circledR}$ \\ 1. Department of Cardiology, Renmin Hospital of Wuhan University, Wuhan 430060, China. \\ 2. Cardiovascular Research Institute, Wuhan University, Wuhan, China. \\ 3. Hubei Key Laboratory of Cardiology, Wuhan, China. \\ 4. Department of Emergency, Renmin Hospital of Wuhan University, Wuhan, China. \\ 5. Department of Pediatrics, Renmin Hospital of Wuhan University, Wuhan, China. \\ 6. Medical Quality Management Office, Renmin Hospital of Wuhan University, Wuhan, China. \\ 7. Department of Medical Affaires, Renmin Hospital of Wuhan University, China. \\ * These authors contributed equally. \\ $\triangle$ Corresponding authors: Dr. Jun Wan, Department of Cardiology, Renmin Hospital of Wuhan University, 238, Jiefang Road, Wuhan 430060, China. Tel.: \\ +86-27-88041911; Fax: +86-27-88042293; E-mail address: wanjun@whu.edu.cn; Dr. Menglong Wang, Department of Cardiology, Renmin Hospital of Wuhan \\ University, 238 Jiefang Road, Wuhan 430060, China. Email address: whuwangmenglong@163.com \\ (C) The author(s). This is an open access article distributed under the terms of the Creative Commons Attribution License (https://creativecommons.org/licenses/by/4.0/). \\ See http://ivyspring.com/terms for full terms and conditions.
}

Received: 2020.10.18; Accepted: 2021.01.04; Published: 2021.02.18

\begin{abstract}
Aim: In other respiratory infectious diseases, obesity may be associated with a poor outcome. For coronavirus disease 2019 (COVID-19), the association between obesity and severity or prognosis requires further analysis.

Methods: This was a retrospective, single-center study. Hospitalized patients were recruited in Renmin Hospital of Wuhan University from January 2, 2020 to February 20, 2020. The data of body mass index (BMI) was obtained from follow-up of surviving patients. According to BMI, normal weight was defined as $18.5-23.9 \mathrm{~kg} / \mathrm{m}^{2}$, overweight as $24.0-27.9 \mathrm{~kg} / \mathrm{m}^{2}$ and obesity as $>28.0 \mathrm{~kg} / \mathrm{m}^{2}$.

Results: A total of 463 patients were enrolled, of which 242 (52.3\%) patients were in the normal weight group; 179 (38.7\%) were in the overweight group; and 42 (9.1\%) were in the obesity group. Compared to the normal group, obese patients were more likely to have a higher heart rate; lower finger oxygen saturation; higher levels of white blood cells, neutrophil counts, basophil counts, intravenous glucose, triacylglycerol, uric acid, alanine aminotransferase, creatine kinase-MB, CD19+ cell counts and percentage; and lower levels of monocyte percentage, high density lipoprotein and CD3+ cell percentage. In addition, the proportions of hypertension ( $21.5 \%$ vs. $42.6 \%)$ and severe+critical illness (47.8 vs. $81.0 \%)$ were significantly higher in the obesity group than those in normal group. However, no significant differences were observed between the normal and obesity groups in critical illness, organ damage and defined endpoint (mechanical ventilation or intensive care unit). Multiple logistic regression showed that obesity increased the risk of developing severe+critical illness (Odd ratio 3.586, 95\% Cl 1.550-8.298, $\mathrm{P}=0.003$ ) in patients with COVID-19, and did not affect the risk of critical illness, organ damage and endpoints. Overweight did not affect the risk of severity, organ damage or endpoint in patients with COVID-19.
\end{abstract}

Conclusion: Obesity may be a risk factor for developing severity in patients with COVID-19.

Key words: coronavirus disease 2019, body mass index, obesity, severity. 


\section{Introduction}

The coronavirus disease 2019 (COVID-19) pandemic, caused by severe acute respiratory syndrome coronavirus 2 (SARS-CoV-2), currently is posing a huge health risk to populations around the world. Many COVID-19 patients have underlying diseases, such as hypertension, diabetes, coronary artery disease, chronic obstructive pulmonary disease (COPD), and liver disease, which contribute to the severity and poor prognosis [1-3]. In an observational study from China, the most common comorbidity was hypertension $(16.9 \%)$, followed by diabetes $(8.2 \%)$, COPD $(1.5 \%)$ and chronic kidney diseases $(21 ; 1.3 \%)$ [4]. In another observational study from New York, USA, the most prevalent comorbidities were hypertension (56.6\%), obesity (41.7\%), diabetes $(33.8 \%)$ and coronary artery disease $(11.1 \%)$ [5].

Obesity is another global epidemic, especially in some western countries [6]. Obesity is associated with increased morbidity of hypertension, other cardiovascular diseases, diabetes and cancers [6-8]. Since hypertension and diabetes increase the risk of severe COVID-19 $[9,10]$, it is necessary to investigate the association between obesity and severe illness or mortality due to COVID-19. Obesity can increase the risk of hospitalization with pneumonia among males [11] but may be independently associated with lower short-term mortality in patients with pneumonia [12]. In other infectious diseases, obesity may be associated with a poor outcome in pandemic H1N1 influenza infection [13]. Therefore, we designed this study to analyze the associations among normal, overweight and obese patients in an enrolled COVID-19 cohort from Wuhan, China. The classification was based on body mass index (BMI). Since obesity rates were relatively low in China and other developing countries, our study may provide an aid to the treatment of obese patients with COVID-19 in these countries.

\section{Methods}

\section{Study design and participants}

This was a retrospective study. The study involving human participants was reviewed and approved by the Institutional Ethics Board of Renmin Hospital of Wuhan University.

Consecutive COVID-19 in-hospital patients were recruited in Renmin Hospital of Wuhan University from January 2, 2020 to February 20, 2020. Renmin Hospital of Wuhan University was a designated hospital during epidemic in Wuhan, which mainly admitted non-mild patients with COVID-19. All patients with COVID-19 were diagnosed with fluorescence reverse transcriptase-polymerase chain reaction (fRT-PCR) assay for SARS-CoV-2 according to the Diagnosis and Treatment of Novel Coronavirus Pneumonia (6th edition) guidelines published by the National Health Commission of China [14]. The data of BMI was obtained from the telephone follow-up of surviving patients and the follow-up time was two weeks after discharge. According to the Chinese-specific cut-offs for general adiposity, BMI $18.5-23.9 \mathrm{~kg} / \mathrm{m}^{2}$ is defined as normal weight, BMI $24.0-27.9 \mathrm{~kg} / \mathrm{m}^{2}$ as overweight and BMI $>28.0 \mathrm{~kg} / \mathrm{m}^{2}$ as general obesity $[15,16]$. Exclusion criteria: patients without BMI data or BMI < 18.5, pregnancy, acute myocardial infarction, malignancy and transplantation. A total of 490 COVID-19 patients had BMI data. After excluding 27 patients based on the exclusion criteria, a total of 463 patients, ultimately, were enrolled. Patients were divided into three groups: normal weight $(n=242)$, overweight $(n=179)$ and obesity $(n=42)$. The strategy of participant enrollment was showed in Figure 1.

According to the Diagnosis and Treatment of Novel Coronavirus Pneumonia (6th edition) guidelines [14], patients were classified as mild/moderate, severe and critical severe. The detailed criteria of classification include: Mild: mild signs or symptoms, without signs of pneumonia; Moderate: general signs or symptoms, imaging shows pneumonia; Severe: Meeting any of the following conditions: 1 . respiratory rate $\geq 30$ beats per minute; 2 . resting $\mathrm{SpO} 2 \leq 93 \%$; 3. arterial $\mathrm{PaO} 2 / \mathrm{FiO} 2 \leq 300$ $\mathrm{mmHg}$; Critical: Meeting any of the following conditions: 1. respiratory failure, needing mechanical

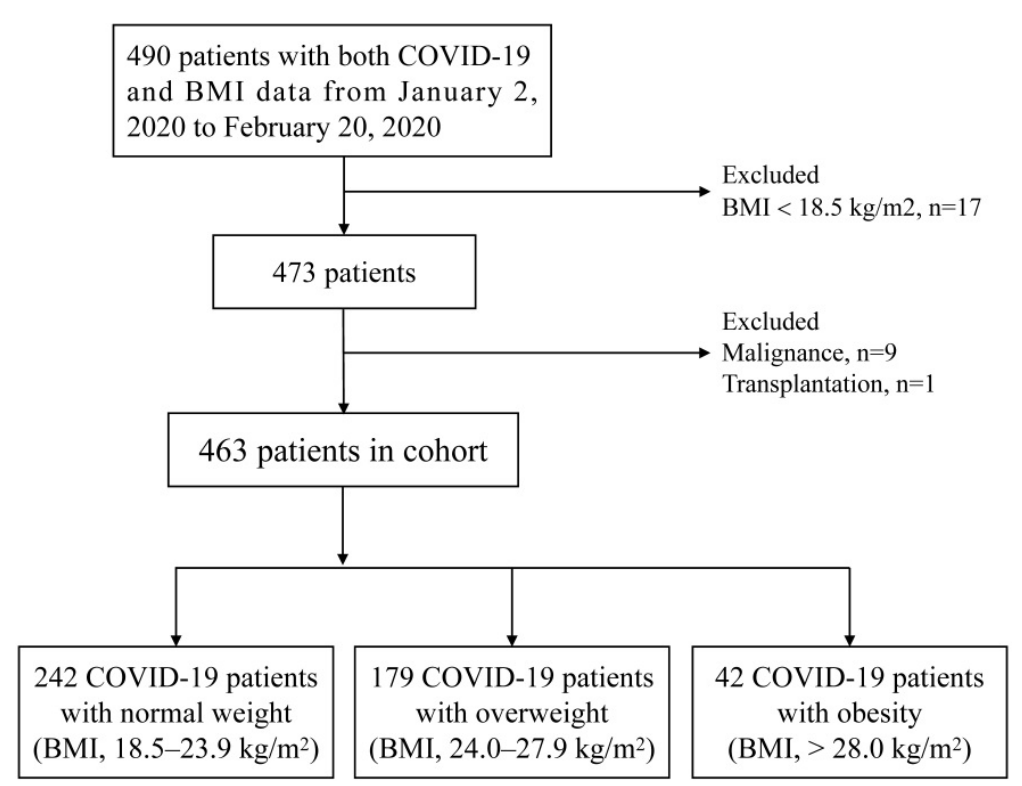

Figure 1. The flowchart showing the strategy of participant enrollment. 
ventilation; 2 . shock; 3 . combined with other organ failure, requiring intensive care. All patients were followed by doctors for 30 days during hospitalization, unless the patients were discharged.

\section{Data collection}

The clinical data (e.g. basic information, initial symptoms, signs at admission, comorbidities, the first laboratory findings, treatment, complications and outcomes) were extracted and obtained by experienced clinicians based on the medical records system of the hospital. And, the first laboratory findings were obtained within three days of admission. Manifestations of lung on computed tomography (CT) were extracted and summarized by integrating the documentation or description in medical charts.

\section{Definition}

Since BMI data (height and weight data) were obtained from the follow-up of survival patients, we defined the primary endpoint as a composite of mechanical ventilation or intensive care unit (ICU) within 30 days. The organ damage was a composite of shock, acute myocardial injury, acute kidney injury or acute liver injury within 30 days of hospitalization. Organ damages or injuries were defined as follows: Acute myocardial injury: Plasma hypersensitivity troponin I (hs-TnI) levels above the upper limit of normal value; Acute liver injury: Alanine aminotransferase (ALT) $\geq 150 \mathrm{U} / \mathrm{L}$; Acute kidney injury: Patients satisfied either of the following conditions: 1) the highest serum creatinine level increased by more than $26.5 \mu \mathrm{mol} / \mathrm{L}(0.3 \mathrm{mg} / \mathrm{dL})$ within 48 hours; or 2) Serum creatinine exceeded the baseline value by 1.5 -fold (confirmed or estimated to occur within 7 days). Shock was considered based on the criteria: Hypotensive patients with failed volume resuscitation were administered vasopressors to maintain blood pressure.

\section{Statistics analysis}

No assumptions were made regarding missing data. SPSS 22.0 software and GraphPad prism 7.0 were used to perform Statistical analysis. The continuous variables were expressed as the median interquartile range $(\mathrm{IQR})$ or mean \pm standard error of the mean (SEM), and the differences between any 2 groups were performed by the Mann-Whitney test or student $t^{\prime}$ test. The categorical variables were presented as number (percentages) and were compared using chi-square tests, or fisher's exact test, if the data was limited. Logistic regression analysis was applied to investigate the association between overweight or obesity and severity, organ damage or endpoint, with the odd ratio (OR) and 95\% confidence interval (95\% CI) being reported. A two-tail p value < 0.05 was considered statistically significant.

\section{Results}

\section{Basic characteristics of COVID-19 patients among the normal, overweight and obesity groups}

A total of 463 patients diagnosed with COVID-19 were enrolled (Figure 1), of which 242 patients $(52.3 \%)$ were divided into the normal weight group, 179 patients (38.7\%) into the overweight group and 42 $(9.1 \%)$ into the obesity group. The median [IQR] ages were $62(49,68)$ years in the normal group, $59(50,67)$ in the overweight group and $63(45,68)$ in the obesity group, and no significant differences were observed among the three groups. With the advancement of BMI, there were no significant differences in the proportion of males $(45.5 \%$ vs. $50.8 \%$ vs. $54.8 \%)$ among the three groups. Similarly, there were no significant differences in the proportions of several initial symptoms among the three groups, including fever, cough, fatigue, chest distress, anorexia, diarrhea and muscle ache. Regarding signs at admission, compared to the normal group, the obesity group had higher heart rate (median, 83 vs. 85 vs. 90 bpm/min) and lower finger oxygen saturation (median, 98 vs. 96 vs. $96 \%)$. However, there were no significant differences among the three groups in respiratory rate and temperature (Table 1).

Regarding comorbidities, the proportion of COVID-19 patients with hypertension $(21.5 \%$ vs. $31.3 \%$ vs. $42.6 \%$ ) increased with the advancement of BMI. There were no significant differences among the three groups in other comorbidities (e.g., diabetes, coronary artery disease, arrhythmia, COPD/asthma, cerebrovascular disease, chronic liver disease and renal disease). Furthermore, the duration from disease onset to admission was also analyzed, and no differences were found among the three groups (Table 1).

\section{First findings of laboratory tests within three days of admission in patients with COVID-19}

We first compared the blood routines among the three groups. Compared to the normal group, the overweight and obesity groups had higher levels of white blood cells (WBC) (median, 4.88 vs. 5.76 vs. 6.49 $\times 10^{9} / \mathrm{L}$ ), neutrophils (median, 3.23 vs. 3.91 vs. 4.34 $\times 10^{9} / \mathrm{L}$ ) and basophilic granulocytes (median, 0.01 vs. 0.01 vs. $0.02 \times 10^{9} / \mathrm{L}$ ). In addition, the levels of C-reactive protein (CRP) (median, 33.1 vs. 41.65 $\mathrm{mg} / \mathrm{L}$ ) and procalcitonin (median, 0.046 vs. 0.06 $\mathrm{ng} / \mathrm{mL}$ ) were also higher in the obesity group than those in the normal weight group, although the 
differences were not significant, possibly because the size of the obesity group was small. These results indicated that the patients in the obesity group had more severe secondary bacterial infections (Table 2).

Compared to the normal group, both the overweight and obesity groups had higher intravenous glucose (median, 5.33 vs. 5.76 vs. 6.29 $\mathrm{mmol} / \mathrm{L}$ ). Triacylglycerol (median, 1.14 vs. 1.34 mmol/L) and uric acid (median, 232 vs. 305 pmol/L) levels were higher and high-density lipoprotein levels (median, 0.97 vs. $0.88 \mathrm{mmol} / \mathrm{L}$ ) were lower in the obesity group than those in the normal weight group. However, no significant differences were observed in low-density lipoprotein and cholesterol. These results may suggest that patients in the obesity group had worse metabolic disorders (Table 2).

With the advancement of BMI, the levels of alanine aminotransferase (median, 20 vs. 31 vs. 33 $\mathrm{U} / \mathrm{L}$ ), aspartate aminotransferase (median, 24 vs. 29 vs. $30 \mathrm{U} / \mathrm{L}$ ), $\mathrm{\gamma}$-glutamyl transferase (median, 22 vs. 33 vs. $43 \mathrm{U} / \mathrm{L}$ ), alkaline phosphatase(median, 59 vs. 64 vs. $65 \mathrm{U} / \mathrm{L}$ ), total bilirubin (median, 9.8 vs. 10.8 vs. $11.2 \mu \mathrm{mol} / \mathrm{L}$ ), direct bilirubin (median, 3.5 vs. 3.9 vs.
$4.2 \mu \mathrm{mol} / \mathrm{L}$ ), lactate dehydrogenase (median, 252 vs. 268 vs. $286 \mathrm{U} / \mathrm{L}$ ) and creatine kinase-MB (median, 0.89 vs. 0.96 vs. $1.12 \mathrm{ng} / \mathrm{mL}$ ) were increased, although no significant differences were observed between the normal and obesity groups in aspartate aminotransferase, alkaline phosphatase, total bilirubin, direct bilirubin and lactate dehydrogenase. These results indicated that the COVID-19 patients with obesity may have more severe organ dysfunction or injury at admission (Table 2).

Compared to the normal group, the obesity group had higher levels of CD19+ cell counts (median, 129 vs. $195 / \mu \mathrm{L}$ ) and CD19+ cell percentage (14.7 vs. $19.1 \%$ ) and lower levels of CD3+ cell percentage $(68.8 \%$ vs. $62.4 \%)$. In addition, the CD4+ cell and CD8+ cell counts were lower in the obesity group, although no significant differences were detected; the limited number of patients in the obesity group may be responsible for this finding. Therefore, a more severe dysfunction of lymphocyte immunity may be present in patients in the obesity group (Table 2).

Table 1. Basic characteristics of patients with COVID-19 among normal, overweight and obesity group

\begin{tabular}{|c|c|c|c|}
\hline Characteristics & Normal $(n=242)$ & Overweight(n=179) & Obesity $(n=42)$ \\
\hline Age, years & $62(49,68)$ & $59(50,67)$ & $63(45,68)$ \\
\hline Male & $110(45.5)$ & $91(50.8)$ & $23(54.8)$ \\
\hline \multicolumn{4}{|l|}{ Initial symptom } \\
\hline Fever & $198(81.8)$ & $149(83.2)$ & $31(73.8)$ \\
\hline Cough & $143(59.1)$ & $110(61.5)$ & $27(64.3)$ \\
\hline Chest distress & $50(20.7)$ & $45(25.1)$ & $7(16.7)$ \\
\hline Dyspnea & $53(21.9)$ & $60(33.5)^{*}$ & $8(19.0)$ \\
\hline Fatigue & $80(33.1)$ & $60(33.5)$ & $12(28.6)$ \\
\hline Muscle ache & $19(7.9)$ & $11(6.1)$ & $3(7.1)$ \\
\hline Anorexia & $23(9.5)$ & 19 (10.6) & $8(19.0)$ \\
\hline Diarrhea & $31(12.8)$ & $20(11.2)$ & $6(14.3)$ \\
\hline \multicolumn{4}{|l|}{ Signs at admission } \\
\hline Temperature ${ }^{A},{ }^{\circ} \mathrm{C}$ & $36.7(36.5,37.3)$ & $36.7(36.4,37.3)$ & $36.8(36.5,37.0)$ \\
\hline Heart rate ${ }^{B}, \mathrm{bpm}$ & $83(78,96)$ & $85(78,94)$ & $90(81,102)^{*}$ \\
\hline Respiratory rate ${ }^{B}, \mathrm{bpm}$ & $20(18,20)$ & $20(18,20)$ & $20(19,21)$ \\
\hline Finger oxygen saturation $C, \%$ & $98(96,99)$ & $96(95,98)$ & $96(95,97.75)^{*}$ \\
\hline \multicolumn{4}{|l|}{ Comorbidity (n, \%) } \\
\hline Hypertension & $52(21.5)$ & $56(31.3)^{*}$ & $18(42.9)^{*}$ \\
\hline Diabetes mellitus & $25(10.3)$ & $22(12.3)$ & $4(9.5)$ \\
\hline Coronary artery disease & $12(5.0)$ & $11(6.1)$ & $4(9.5)$ \\
\hline Arrythmia & $8(3.3)$ & $2(1.1)$ & $1(2.4)$ \\
\hline COPD/Asthma & $11(4.5)$ & $3(1.7)$ & 0 \\
\hline Cerebrovascular disease & $5(2.1)$ & $2(1.1)$ & $2(4.5)$ \\
\hline Chronic kidney disease & $6(2.5)$ & $2(1.1)$ & 0 \\
\hline Chronic liver disease & $7(2.9)$ & $7(3.9)$ & $1(2.4)$ \\
\hline Only one comorbidity & $55(22.7)$ & $52(29.1)$ & $12(28.6)$ \\
\hline$\geq 2$ comorbidities & $32(13.2)$ & $25(14.0)$ & $10(23.8)$ \\
\hline Duration before admission & $10(7,13)$ & $11(8,15)$ & $11(8,14)$ \\
\hline
\end{tabular}


Table 2. First laboratory findings of COVID-19 patients within 3 days of admission among normal, overweight and obesity group

\begin{tabular}{|c|c|c|c|}
\hline & Normal (n=242) & Overweight (n=179) & Obesity $(n=42)$ \\
\hline \multicolumn{4}{|l|}{ Blood routine $^{A}$} \\
\hline White blood cell count, $\times 10^{9} / \mathrm{L}$ & $4.88(3.81,6.49)$ & $5.76(4.42,7.2)^{*}$ & $6.49(4.27,7.79)^{*}$ \\
\hline Neutrophil percentage, $\%$ & $66.1(56.2,75.8)$ & $68.7(60.9,79.3)^{*}$ & $71.7(62.4,79.0)$ \\
\hline Neutrophil count, $\times 10^{9} / \mathrm{L}$ & $3.23(2.31,4.63)$ & $3.91(2.7,5.38)^{*}$ & $4.34(2.74,6.32)^{*}$ \\
\hline Lymphocyte percentage, \% & $23.3(15.4,31.8)$ & $19.6(12.3,28.3)^{*}$ & $20.2(13.4,28.7)$ \\
\hline Lymphocyte count, $\times 109 / \mathrm{L}$ & $1.08(0.81,1.46)$ & $1.12(0.79,1.53)$ & $1.12(0.89,1.65)$ \\
\hline Monocyte percentage, $\%$ & $8.6(6.3,10.9)$ & $8.5(6.1,10.4)$ & $7.6(5.3,8.7)^{*}$ \\
\hline Monocyte count, ×109/L & $0.42(0.31,0.56)$ & $0.45(0.35,0.61)$ & $0.45(0.34,0.57)$ \\
\hline Basophil count, ×109/L & $0.01(0.01,0.02)$ & $0.01(0.01,0.03)^{*}$ & $0.02(0.01,0.03)^{*}$ \\
\hline Eosinophil count, ×109/L & $0.01(0,0.06)$ & $0.03(0,0.08)$ & $0.01(0,0.09)$ \\
\hline Blood platelet count, $\times 109 / \mathrm{L}$ & $214(160,273)$ & $230(185,286)^{*}$ & $230(168,308)$ \\
\hline \multicolumn{4}{|l|}{ Inflammation } \\
\hline C-reactive protein ${ }^{B}, \mathrm{mg} / \mathrm{L}$ & $33.1(6.35,70.15)$ & $27(6.65,60.85)$ & $41.65(9.28,70.5)$ \\
\hline hsC-reactive protein ${ }^{C}, \mathrm{mg} / \mathrm{L}$ & $5(5,5)$ & $5(5,5)$ & $5(5,5)$ \\
\hline Procalcitonin ${ }^{D}, \mathrm{ng} / \mathrm{mL}$ & $0.046(0.028,0.096)$ & $0.051(0.036,0.082)$ & $0.06(0.04,0.08)$ \\
\hline Intravenous glucose $E, \mathrm{mmol} / \mathrm{L}$ & $5.33(4.79,6.61)$ & $5.76(5.1,7.79)^{*}$ & $6.29(5.13,7.15)$ \\
\hline \multicolumn{4}{|l|}{ Blood lipid $F$} \\
\hline Total cholesterol, mmol/L & $3.8(3.26,4.47)$ & $3.75(3.33,4.43)$ & $3.85(3.33,4.40)$ \\
\hline Triglyceride, mmol/L & $1.14(0.9,1.52)$ & $1.19(0.97,1.68)$ & $1.34(1.09,2.16)^{*}$ \\
\hline Low density lipoprotein, mmol/L & $2.27(1.85,2.91)$ & $2.45(1.94,2.88)$ & $2.46(2.01,2.97)$ \\
\hline High density lipoprotein, $\mathrm{mmol} / \mathrm{L}$ & $0.97(0.81,1.14)$ & $0.87(0.77,1.05)^{*}$ & $0.88(0.72,1.01)^{*}$ \\
\hline \multicolumn{4}{|l|}{ Blood electrolyte $F$} \\
\hline $\mathrm{K}+$ & $3.9(3.6,4.4)$ & $3.9(3.6,4.2)$ & $4.0(3.6,4.3)$ \\
\hline $\mathrm{Na}+$ & $141(138,144)$ & $141(138,144)$ & $141(137,144)$ \\
\hline \multicolumn{4}{|l|}{ Liver function } \\
\hline Alanine aminotransferase ${ }^{G}, \mathrm{U} / \mathrm{L}$ & $20(15,32)$ & $31(20,54)^{*}$ & $33(21,47)^{*}$ \\
\hline Aspartate aminotransferase ${ }^{G}, \mathrm{U} / \mathrm{L}$ & $24(19,34)$ & $29(21,43)^{*}$ & $30(22,42)$ \\
\hline Y-glutamyltransferase ${ }^{H}, \mathrm{U} / \mathrm{L}$ & $22(15,35)$ & $33(22,57)^{*}$ & $43(25,64)^{*}$ \\
\hline Alkaline phosphatase ${ }^{H}, \mathrm{U} / \mathrm{L}$ & $59(49,70)$ & $64(54,79)^{*}$ & $65(54,84)$ \\
\hline Total bilirubin ${ }^{H}, \mu \mathrm{mol} / \mathrm{L}$ & $9.8(7.6,12.6)$ & $10.8(8.1,14.2)^{*}$ & $11.2(8.8,14.8)$ \\
\hline Direct bilirubin ${ }^{H}, \mu \mathrm{mol} / \mathrm{L}$ & $3.5(2.5,4.8)$ & $3.9(2.9,5)^{*}$ & $4.2(2.9,5.4)$ \\
\hline \multicolumn{4}{|l|}{ Kidney Function ${ }^{I}$} \\
\hline Creatinine, $\mu \mathrm{mol} / \mathrm{L}$ & $60(49,72)$ & $61(50,72)$ & $65(50,76)$ \\
\hline Blood urea nitrogen, $\mathrm{mmol} / \mathrm{L}$ & $4.29(3.41,5.59)$ & $4.4(3.58,5.59)$ & $4.5(3.95,5.72)$ \\
\hline Blood uric acid, $\mu \mathrm{mol} / \mathrm{L}$ & $232(187,298)$ & $253(211,319)^{*}$ & $305(231,357)^{*} \#$ \\
\hline $\mathrm{eGFR}, \mathrm{mL} / \mathrm{min}$ & $98.4(90.0,106.9)$ & $98.9(92.0,108.3)$ & $97.3(85.6,112.1)$ \\
\hline \multicolumn{4}{|l|}{ Myocardial injury } \\
\hline Creatine kinase $J, \mathrm{ng} / \mathrm{mL}$ & $57(37,87)$ & $59(39,106)$ & $64(42,115)$ \\
\hline Creatine kinase-MB ${ }^{K}, \mathrm{ng} / \mathrm{mL}$ & $0.89(0.54,1.35)$ & $0.96(0.66,1.26)$ & $1.12(0.76,2.2)^{*}$ \\
\hline Lactate dehydrogenase $^{L}, \mathrm{U} / \mathrm{L}$ & $252(203,310)$ & $268(217,340)^{*}$ & $286(208,356)$ \\
\hline Myoglobin ${ }^{M}, \mathrm{~g} / \mathrm{L}$ & $36.54(25.26,58.8)$ & $40.03(27.92,61.3)$ & $45.04(30.57,80.48)$ \\
\hline Hypersensitive troponin $\mathrm{I}^{N}, \mathrm{ng} / \mathrm{mL}$ & $0.006(0.006,0.009)$ & $0.006(0.006,0.008)$ & $0.006(0.006,0.009)$ \\
\hline NTpro-BNP ${ }^{o}, \mathrm{pg} / \mathrm{ml}$ & $114.7(47.3,235.2)$ & $105.1(34.6,235.3)$ & $152.7(61.5,370.1)$ \\
\hline \multicolumn{4}{|l|}{ Coagulation function ${ }^{P}$} \\
\hline D-Dimer, mg/L & $0.62(0.36,1.44)$ & $0.63(0.38,1.36)$ & $0.72(0.4,2.67)$ \\
\hline Prothrombin time activity, $\%$ & $84.7(75.9,95)$ & $84.7(77.3,93.3)$ & $85.4(82.2,91.4)$ \\
\hline APTT, sec & $28.3(26.3,31.3)$ & $27.8(25.6,30.2)^{*}$ & $27.7(25.5,31.2)$ \\
\hline International normalized ratio & $1.03(0.97,1.08)$ & $1.03(0.97,1.07)$ & $1.03(0.99,1.05)$ \\
\hline Thrombin time, sec & $17.6(16.7,18.4)$ & $17.6(16.6,18.5)$ & $17.4(16.8,18.2)$ \\
\hline Prothrombin time, sec & $12(11.4,12.6)$ & $11.95(11.425,12.5)$ & $12(11.6,12.2)$ \\
\hline Antithrombin III activity, \% & $89.9(82.2,97.8)$ & $88.8(81.6,97.8)$ & $87.6(82.1,95.9)$ \\
\hline Fibrin degradation products, $\mathrm{mg} / \mathrm{L}$ & $2.28(0.94,4.99)$ & $1.93(0.98,5.38)$ & $2.8(1.81,7.35)$ \\
\hline Fibrinogen, $\mathrm{g} / \mathrm{L}$ & $4.56(3.42,5.46)$ & $4.56(3.73,5.7)$ & $4.69(3.52,5.87)$ \\
\hline \multicolumn{4}{|l|}{ Cellular immunity $Q$} \\
\hline CD16+56+ cell percentage, $\%$ & $13.1(8.3,21.5)$ & $12.5(8.5,18.8)$ & $16.4(10.1,21.3)$ \\
\hline CD16+56+ cell counts, $/ \mu \mathrm{L}$ & $119(71,180)$ & $117(77,175)$ & $159(104,193)$ \\
\hline CD19+ cell percentage, $\%$ & $14.7(11.0,19.3)$ & $17.5(13.2,21.6)^{*}$ & $19.1(15.2,21.3)^{*}$ \\
\hline CD19+ cell counts, $/ \mu \mathrm{L}$ & $129(9,184)$ & $159(106,247)^{*}$ & $195(124,280)^{*}$ \\
\hline CD3+ cell percentage, $\%$ & $68.8(58.1,75.6)$ & $67.2(58.0,71.9)$ & $62.4(53.4,69.5)^{*}$ \\
\hline CD3+ cell counts, $/ \mu \mathrm{L}$ & $623(427,882)$ & $659(441,927)$ & $628(424,858)$ \\
\hline CD4+ cell percentage, $\%$ & $39.4(32.0,46.5)$ & $40.4(33.8,46.1)$ & $39.7(33.4,45.5)$ \\
\hline CD4 cell counts, $/ \mu \mathrm{L}$ & $375(249,513)$ & $420(251,576)$ & $359(241,535)$ \\
\hline CD8+ cell percentage, $\%$ & $23.8(17.2,30.1)$ & $21.5(16.5,28.0)$ & $18.3(14.0,27.2)$ \\
\hline CD8+ cell counts, $/ \mu \mathrm{L}$ & $231(128,335)$ & $207(132,315)$ & $212(114,321)$ \\
\hline CD4/CD8 ratio & $1.67(1.21,2.41)$ & $1.8(1.32,2.7)$ & $2.14(1.38,3.17)$ \\
\hline \multicolumn{4}{|l|}{ Arterial blood gas analysis $R$} \\
\hline PH value & $7.42(7.39,7.46)$ & $7.43(7.37,7.46)$ & $7.44(7.38,7.46)$ \\
\hline
\end{tabular}




\begin{tabular}{|c|c|c|c|}
\hline & Normal $(n=242)$ & Overweight $(n=179)$ & Obesity $(n=42)$ \\
\hline Partial pressure of oxygen, $\mathrm{mmHg}$ & $88(70,112)$ & $83(70,109)$ & $78(55,87)$ \\
\hline Arterial oxygen saturation, $\%$ & $97(94,98)$ & $96(94,98)$ & $95(90,97)$ \\
\hline Arterial lactate acid, mmol/L & $1.9(1.5,2.5)$ & $1.9(1.4,2.6)$ & $2.2(1.6,2.6)$ \\
\hline
\end{tabular}

Values are Medium (IQR) unless stated otherwise. Continuous variables were compared by the Mann-Whitney test. * $p<0.05$ vs. Normal weight group; \# p $<0.05$ vs.

Overweight group. Abbreviation: hsC-reactive protein, high-sensitive reactive protein; eGFR, estimated glomerular filtration rate; NTpro-BNP, N-terminal pro-Brain Natriuretic Peptide; APTT, activated partial thromboplastin time. The total number of patients with available data in different groups (n1, n2 and n3 indicates the number in normal weight, overweight and obesity group, respectively): $A: \mathrm{n} 1=237, \mathrm{n} 2=175, \mathrm{n} 3=42 ; B: \mathrm{n} 1=199, \mathrm{n} 2=155, \mathrm{n} 3=36 ; C: \mathrm{n} 1=202, \mathrm{n} 2=159, \mathrm{n} 3=36 ; D: \mathrm{n} 1=218, \mathrm{n} 2=170, \mathrm{n} 3=39 ; E$ : n1=238, n2=179, n3=42; F: n1=230, n2=173, n3=42; F: n1=238, n2=178, n3=41; G: n1=238, n2=179, n3=42; H: n1=232, n2=176, n3=42; I: n1=238, n2=179, n3=42; J: n1=232, n2=173, n3=41; K: n1=200, n2=140, n3=37; $L: \mathrm{n} 1=232, \mathrm{n} 2=171, \mathrm{n} 3=41 ; M: \mathrm{n} 1=199, \mathrm{n} 2=140, \mathrm{n} 3=36 ; N: \mathrm{n} 1=201, \mathrm{n} 2=145, \mathrm{n} 3=36 ; \boldsymbol{O}: \mathrm{n} 1=175, \mathrm{n} 2=130, \mathrm{n} 3=31 ; P: \mathrm{n} 1=215, \mathrm{n} 2=161$, n3=39; $Q: \mathrm{n} 1=198, \mathrm{n} 2=144, \mathrm{n} 3=33 ; R: \mathrm{n} 1=105, \mathrm{n} 2=82, \mathrm{n} 3=17$.

Table 3. Lung CT findings of COVID-19 patients at admission among normal, overweight and obesity group

\begin{tabular}{llll}
\hline Features of lung CT & \multicolumn{1}{l}{ Normal $(\mathbf{n = 1 2 9})$} & Overweight $(\mathbf{n}=\mathbf{8 6})$ & Obesity $(\mathbf{n}=\mathbf{1 8})$ \\
\hline Pneumonia & $129(100)$ & $85(98.8)$ & $18(100)$ \\
Unilateral lung & $13(10.1)$ & $2(2.3)$ & 0 \\
Bilateral lung & $116(89.9)$ & $83(96.5)$ & $18(100)$ \\
Ground-glass opacity & $104(80.6)$ & $71(82.6)$ & $14(77.8)$ \\
reticular/linear & $35(27.1)$ & $25(29.1)$ & $5(27.8)$ \\
Air bronchogram & $15(11.6)$ & $11(12.8)$ & $4(22.2)$ \\
Consolidation shadow & $15(11.6)$ & $22(25.6)^{*}$ & $5(27.8)$ \\
\hline
\end{tabular}

Values are number (percentage). Categorical variables were compared by chi-square tests, or fisher's exact test. ${ }^{*} \mathrm{p}<0.05$ vs. Normal weight group; \# $\mathrm{p}<0.05$ vs. Overweight group. Abbreviation: CT, computerized tomography.

Table 4. Treatment, typing, complication and endpoint of COVID-19 patients among normal, overweight and obesity group

\begin{tabular}{llll}
\hline & $\begin{array}{l}\text { Normal } \\
(\mathbf{n = 2 4 2})\end{array}$ & $\begin{array}{l}\text { Overweight } \\
\text { (n=179) }\end{array}$ & $\begin{array}{l}\text { Obesity } \\
\text { (n=42) }\end{array}$ \\
\hline $\begin{array}{l}\text { Treatment } \\
\text { Antiviral therapy }\end{array}$ & $237(97.9)$ & $170(95.0)$ & $41(97.6)$ \\
Antibacterial therapy & $200(82.6)$ & $141(78.8)$ & $37(88.1)$ \\
Antifungal therapy & $5(2.7)$ & $2(1.1)$ & $1(2.4)$ \\
Glucocorticoid therapy & $106(43.8)$ & $72(40.2)$ & $20(47.6)$ \\
Immunoglobulin therapy & $129(53.3)$ & $79(44.1)$ & $25(59.5)$ \\
Traditional Chinese & $187(77.3)$ & $128(71.5)$ & $33(78.6)$ \\
medicine & & & \\
Oxygen therapy & $213(88.0)$ & $161(89.9)$ & $37(88.1)$ \\
Typing & $102(42.2)$ & $61(34.1)$ & $8(19.0)^{*}$ \\
Mild-moderate & $119(49.2)$ & $99(55.3)$ & $27(64.3)$ \\
Severe & $21(8.7)$ & $19(10.6)$ & $7(16.7)$ \\
Critical & $32(13.2)$ & $21(11.7)$ & $10(23.8) \#$ \\
Complication & $4(1.7)$ & $3(1.7)$ & $2(4.8)$ \\
Shock & $16(6.6)$ & $8(4.5)$ & $4(9.5)$ \\
Acute myocardial injury & $1(0.4)$ & $2(1.1)$ & 0 \\
Acute kidney injury & $15(6.2)$ & $10(5.6)$ & $4(9.5)$ \\
Acute liver injury & $21(8.68)$ & $19(10.6)$ & $5(11.9)$ \\
Endpoint & & &
\end{tabular}

Values are number (percentage). Categorical variables were compared by chi-square tests, or fisher's exact test. ${ }^{*} \mathrm{p}<0.05$ vs. Normal weight group; $\# \mathrm{p}<0.05$ vs. Overweight group. Endpoint was a composite of mechanical ventilation or admission of intensive care unit, after all patients were followed for 30 days.

\section{Manifestations of chest CT at admission and treatment during hospitalization in COVID-19 patients}

Regarding lung CT findings at admission, we found that no significant differences were detected among three groups in several manifestations, including unilateral pneumonia, bilateral pneumonia, ground-glass opacity, reticular/linear and air bronchogram. Compared to the normal group, the overweight group had more patients with consolidation shadows in lung CT (Table 3).
Regarding treatment, there were no differences among the three groups in major treatment regimens, such as antiviral therapy, antibacterial therapy, antifungal therapy, glucocorticoids, immunoglobulin, traditional Chinese medicine and oxygen therapy (Table 4).

\section{The association between overweight or obesity and severity, organ injury or designed endpoint}

Compared to the normal weight group, the proportion of mild-moderate patients (42.2 vs. $19.0 \%$ ) was decreased in the obesity group. The proportions of severe+critical illness ( 47.8 vs. $81.0 \%$ ) were higher in the obesity group than those in the normal weight group. No significant differences were observed between the normal and overweight groups. Compared to the overweight group, the obesity group had a higher proportion of patients with complications (11.7 vs. $23.8 \%$ ), while, no significant differences were observed between normal group and obesity group. The designed endpoint was a composite of mechanical ventilation or ICU admission. After every patient was followed for 30 days, the differences were not significant among three groups in the endpoint (Table 4).

After adjusting for age, sex, comorbidity (e.g., hypertension, diabetes, coronary artery disease, arrhythmia, cerebrovascular disease, COPD, asthma, chronic renal disease and chronic liver disease), multivariate logistic regression analysis revealed that COVID-19 patients with obesity were more likely to develop severe+critical illness (Odd ratio 3.586, 95\% CI 1.550-8.298, $\mathrm{P}=0.003$ ). However, the association between obesity and the designed endpoint, critical illness or organ damage was not significant. In addition, overweight was not found to be significantly associated with critical illness, severe+critical illness, organ damage and primary endpoint (Table 5).

\section{Depletion of CD4+ cells may contribute to developing severe illness in the subgroup of COVID-19 patients with obesity}

In the subgroup of COVID-19 patients with obesity, compared to the nonsevere group, the levels of CD4 were significantly decreased in the severe 
group. Similar decreased trends in CD3+ cells, CD8+ cells, CD4+/CD8+ ratio, CD19+ cells and lymphocytes and, in turn, increased trends in WBC, neutrophils and CRP were observed, while the differences were not significant. If the size of the obesity group was increased, these trends may be significant. The results indicated that depletion of CD4+ T cells may be responsible for the development of severe illness in the subgroup of COVID-19 patients with obesity (Figure 2A-L).

Table 5. The association between obesity or overweight and severity, organ damage, or endpoint in patients with COVID-19

\begin{tabular}{|c|c|c|c|}
\hline & Odd Ratio & $95 \% \mathrm{CI}$ & P value \\
\hline \multicolumn{4}{|c|}{ Critical illness } \\
\hline \multicolumn{4}{|c|}{ Model 1} \\
\hline Normal & - & - & - \\
\hline Overweight & 1.250 & $0.650-2.401$ & 0.504 \\
\hline Obesity & 2.105 & $0.833-5.317$ & 0.116 \\
\hline \multicolumn{4}{|l|}{ Model 2} \\
\hline Overweight & 1.240 & $0.629-2.445$ & 0.534 \\
\hline Obesity & 1.973 & $0.744-5.231$ & 0.172 \\
\hline \multicolumn{4}{|c|}{ Severe + Critical illness } \\
\hline \multicolumn{4}{|c|}{ Model 1} \\
\hline Overweight & 1.409 & 0.944-2.104 & 0.093 \\
\hline Obesity & 3.096 & $1.376-6.970$ & $0.006^{*}$ \\
\hline \multicolumn{4}{|l|}{ Model 2} \\
\hline Overweight & 1.443 & $0.953-2.185$ & 0.083 \\
\hline Obesity & 3.586 & $1.550-8.298$ & $0.003^{*}$ \\
\hline \multicolumn{4}{|c|}{ Organ damage } \\
\hline \multicolumn{4}{|c|}{ Model 1} \\
\hline Overweight & 0.872 & $0.485-1.570$ & 0.649 \\
\hline Obesity & 2.051 & $0.920-4.571$ & 0.079 \\
\hline \multicolumn{4}{|l|}{ Model 2} \\
\hline Overweight & 0.894 & $0.490-1.634$ & 0.716 \\
\hline Obesity & 2.005 & $0.875-4.593$ & 0.100 \\
\hline \multicolumn{4}{|l|}{ Endpoint } \\
\hline \multicolumn{4}{|l|}{ Model 1} \\
\hline Overweight & 1.250 & $0.650-2.401$ & 0.504 \\
\hline Obesity & 1.422 & $0.505-4.006$ & 0.505 \\
\hline \multicolumn{4}{|l|}{ Model 2} \\
\hline Overweight & 1.231 & $0.622-2.434$ & 0.551 \\
\hline Obesity & 1.299 & $0.440-3.838$ & 0.636 \\
\hline \multicolumn{4}{|c|}{$\begin{array}{l}\text { Endpoint was a composite of mechanical ventilation or admission of intensive care } \\
\text { unit, after all patients were followed for } 30 \text { days. Model 1: Univariate regression } \\
\text { analysis. Model 2: Multivariate regression analysis with adjusting for sex, age and } \\
\text { comorbidities (e.g. hypertension, diabetes, coronary artery disease, arrhythmia, } \\
\text { cerebrovascular disease, COPD, asthma, chronic renal disease and chronic liver } \\
\text { disease). }\end{array}$} \\
\hline
\end{tabular}

\section{Discussion}

Our present study found that obese patients had an increased risk of developing severity in patients with COVID-19. Obese patients were more likely to have hypertension, as well as severe secondary infection, metabolic disorder, organ dysfunction, and disorder of cellular immunity.

Obesity is another global health crisis. In 2012, the prevalence of obesity in adults was $11.9 \%$ in China and has increased rapidly from 2002 to 2012 [17]. In 2013 , the proportions of adults with BMI $\geq 25.0 \mathrm{~kg} / \mathrm{m}^{2}$ have increased to $36.9 \%$ in adult men and $38 \%$ in women, worldwide [18]. During the COVID-19 pandemic, obesity has become one of the most prevalent comorbidities, especially in the USA [5]. Obesity can cause damage to expiratory reserve volume, functional capacity and respiratory system compliance, which may be associated with the aggravation of disease [19]. In the epidemic of HIN1 influenza virus, several studies analyzing the effect of obesity revealed that obesity is strongly associated with severe illness [20-22]. Additionally, there was a large fraction of obese patients among the patients admitted into the ICU [20, 21]. In our study, we found that obesity was associated with an increased risk of developing severity in patients with COVID-19. The conclusion was consistent with the reports from Cai et al. [23] in Shenzhen, China. In addition, Haywood et al. [24] concluded that obesity was a risk factor for hospital admission, suggesting that obese patients needed more hospital care. In our study, we found that obesity did not have an obvious effect on the development of endpoint or organ damage. In a cohort of COVID-19 patients from New York, Palaiodimos et al. [25] indicated that severe obesity $\left(\right.$ BMI $\left.>35.0 \mathrm{~kg} / \mathrm{m}^{2}\right)$ was independently associated with higher in-hospital mortality. In another prospective observational cohort study [26], obesity was also associated with higher in-hospital mortality. The lack of BMI data for patients who died and the limited size of the obesity group may be responsible for the negative results. In addition, the proportion of severe obesity was low in our study, which may be due to the relatively low rate of severe obesity in China [27]. Since severe patients with COVID-19 are associated with poor prognosis, we indicated that COVID-19 patients with obesity may be more likely to develop poor outcomes. Obese patients with COVID-19 may need more attention during hospitalization, even in countries with low obesity rates.

Obese patients are more likely to have diabetes mellitus, hypertension and other cardiovascular diseases [6-8]. Comorbidities including hypertension and diabetes mellitus are associated with mortality in patients with COVID-19 [10, 28, 29]. In addition, complications including shock, acute heart injury, kidney injury and liver injury are commonly observed in severe or critical patients with COVID-19 and can also increase the risk of developing severe illness and poor outcomes [30,31]. In our study, the obesity group had a higher proportion of patients with preexisting diseases; in particular, patients with hypertension. In addition, organ dysfunction was more serious in the obesity group than that in the normal weight group at admission. These characteristics may be responsible for the higher proportions of severe+critical 
COVID-19 in the obesity group. Moreover, obese patients with COVID-19 were more likely to have secondary bacterial infection and relatively high levels of CRP, which also contributed to the development of critical illness. A previous study has indicated that chronic inflammation was present in obese patients [32]. The co-existing inflammation may have aggravated the secondary infection in obese patients with COVID-19.

Obese patients with COVID-19 had higher levels of fasting blood glucose, triacylglycerol and uric acid and lower levels of high-density lipoprotein, indicating that obese patients with COVID-19 were more likely to have metabolic disorder. Costa et al. [33] has suggested that metabolic disorder may be a risk factor for affecting the progression and prognosis of COVID-2019.

Changes in peripheral lymphocyte subsets have been observed in COVID-19 patients [34]. SARS-CoV-2 infection decreased the peripheral levels of CD3+ cells, CD4+ cells and CD8+ cells, which were further reduced in severe illness [35, 36]. Wang et al. [36] also found that the levels of CD19+ cells, not CD16+56+ cells, were lower in severe cases of
COVID-19 than those in mild cases. In our study, we found that percentages of CD3+ cells were lower in the obesity group than those in the normal weight group. The counts of CD4+ cells and CD8+ cells were also decreased in the obesity group, while the differences were not significant. The reductions of these cells may contribute to the high percentages of severe and critical COVID-19 in obese patients. In addition, we showed that the levels of CD19+ B cells were higher in obese patients. Although B cells help the body to generate antibody, they can also promote inflammation in obesity [37]. B cells have been recognized as having an important effect on the control of hepatitis B virus [38]. Therefore, more severe adaptive immune disorders may exist in obese patients with COVID-19, and the detailed roles of CD19+ B cells will require further studies in the future. In the obesity subgroup, compared to the nonsevere patients, peripheral levels of CD4+ cells were decreased in severe COVID-19 patients. The results suggested that depletion of CD4+ cells was associated with the increased risk of progression of COVID-19 in the obesity subgroup.

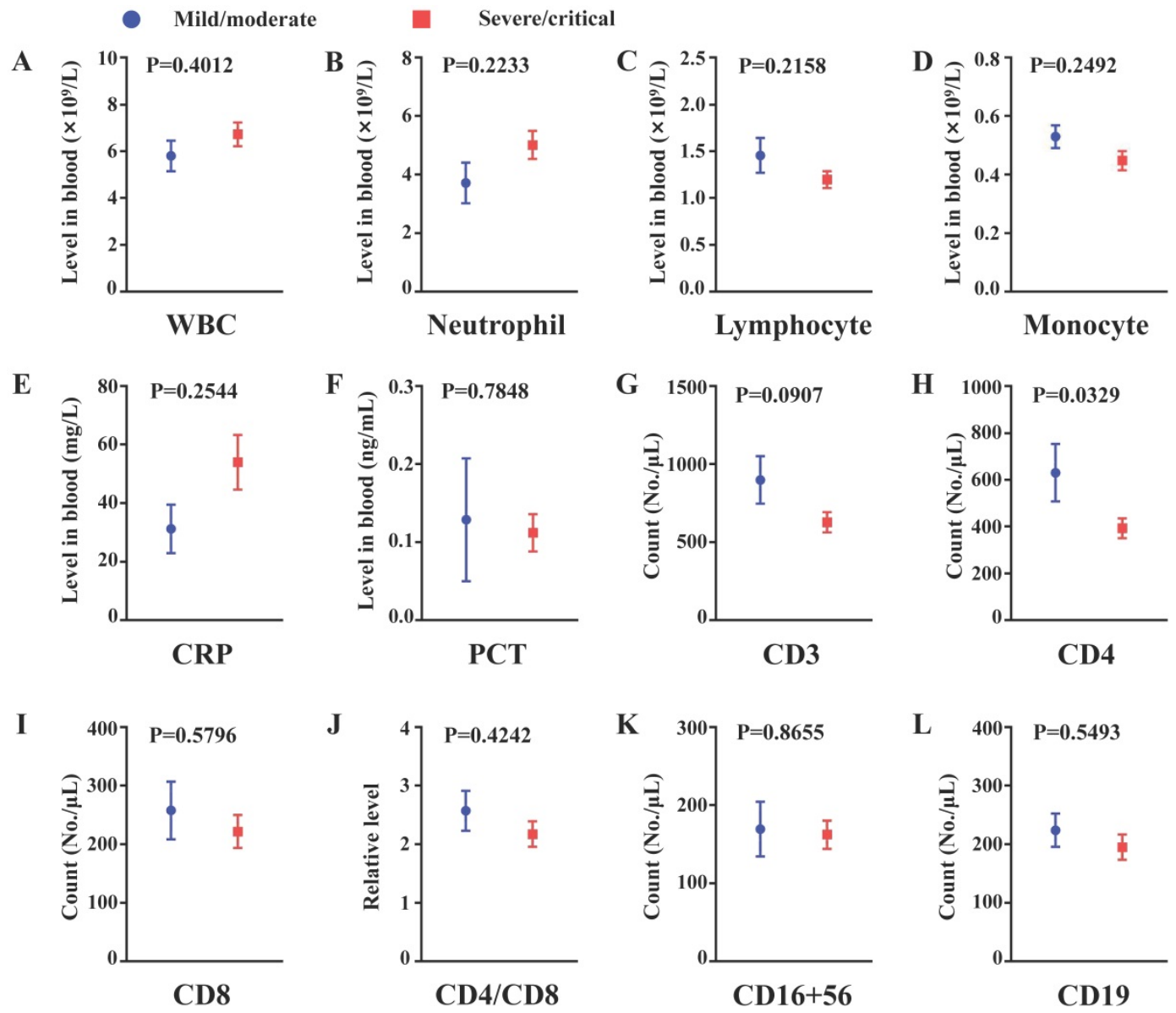

Figure 2. The comparison of inflammation and cellular immunity between non-severe and severe patients from obesity subgroup. (A) White blood cell count (B) Neutrophil count; (C) Lymphocyte count; (D) Monocyte count; (E) C-reactive protein level in peripheral blood; (F) Procalcitonin level in peripheral blood; (G) CD3+ cell count; (H) CD4+ cell count; (I) CD8+ cell count; (J) Relative CD4/CD8 level; (K) CD16+56+ cell count; (L) CD19+ cell count. $n=8$ in the mild/moderate group, $n=27-34$ in the severe/critical group. Data was presented by mean $\pm S E M$ and compared using student $t$ ' test, $p<0.05$ was considered significantly different. 


\section{Limitations}

First, the BMI data were obtained from the follow-up of survival patients and the follow-up time was two weeks after discharge. This gave rise to several potential concerns: the accuracy of the BMI data measurement could be questionable; the BMI was not measured upon the COVID-19 diagnosis but rather was collected at 14-day follow-up, BMI at this point might not be the same as the BMI during in-hospital period, therefore, the BMI data might be either underestimated or overestimated, especially for those whose BMI was at the borderline; some of the most critical patients who did not survive from COVID-19 were not included in this study. Second, the size of the obesity group in our study was quite small. Third, only a small number of patients had data regarding interleukins, interferon $\gamma$, and other cytokines, all of which would help to analyze the role of the inflammatory storm in obese patients with COVID-19.

\section{Conclusion}

Obese patients with COVID-19 were more likely to have hypertension, secondary infection, organ dysfunction, metabolic disorders and dysfunction of lymphocyte immunity. Obesity was associated with an increased risk of developing severe/critical illness in patients with COVID-19.

\section{Acknowledgements}

We acknowledge Renmin Hospital of Wuhan University, which offered enough support for our study.

\section{Data Availability}

All data that support the findings in this study are available from the corresponding author upon reasonable request.

\section{Ethical approval}

The study involving human participants was reviewed and approved by the Institutional Ethics Board of Renmin Hospital of Wuhan University.

\section{Funding}

This study was supported by the emergency technical project for COVID-19 from the Wuhan Science and Technology Bureau (No. 20200202010016).

\section{Informed consent}

Written informed consent from the participants' legal guardian/next of kin was not required to participate in this retrospective study in accordance with the national legislation and the institutional requirements.

\section{Author Contributions}

M. Wang and J. Wan designed the study. M. Wang, M-X. Liu, B. Shen, H. He and J. Wan had full access to all of the data in the study. J. Zhang, M. Wang, M. Zhao, J. Liu and Y. Xu analyzed the data. J. Zhang wrote the original manuscript. J. Zhang, B. Shen and $\mathrm{H}$. He performed the revised manuscript. J. Ye, Z. Wang, D. Ye, W. Pan, S. Xu, Y. Feng, Z. Luo, D. $\mathrm{Li}$ and J. Liu performed the critical revision of the manuscript for important intellectual content. The final version of the manuscript was approved by all the authors.

\section{Competing Interests}

The authors have declared that no competing interest exists.

\section{References}

1. Zhao M, Wang M, Zhang J, et al. Advances in the relationship between coronavirus infection and cardiovascular diseases. Biomed Pharmacother. 2020; 127: 110230 .

2. Zhao M, Wang M, Zhang J, et al. Comparison of clinical characteristics and outcomes of patients with coronavirus disease 2019 at different ages. Aging (Albany NY). 2020; 12: 10070-10086.

3. Zhang J, Wang M, Zhao M, et al. The Clinical Characteristics and Prognosis Factors of Mild-Moderate Patients With COVID-19 in a Mobile Cabin Hospital: A Retrospective, Single-Center Study. Front Public Health. 2020; 8: 264.

4. Guan W, Liang W, Zhao Y, et al. Comorbidity and its impact on 1590 patients with COVID-19 in China: a nationwide analysis. Eur Respir J. 2020; 55: 2000547.

5. Richardson S, Hirsch JS, Narasimhan M, et al. Presenting Characteristics, Comorbidities, and Outcomes Among 5700 Patients Hospitalized With COVID-19 in the New York City Area. JAMA. 2020; 323: 2052.

6. Hales CM, Carroll MD, Fryar CD, Ogden CL. Prevalence of Obesity Among Adults and Youth: United States, 2015-2016. NCHS Data Brief. 2017; p: 1-8.

7. Nguyen T, Lau DCW. The Obesity Epidemic and Its Impact on Hypertension. Can J Cardiol. 2012; 28: 326-333.

8. Khaodhiar L, McCowen KC, Blackburn GL. Obesity and its comorbid conditions. Clin Cornerstone. 1999; 2: 17-31.

9. Guo W, Li M, Dong Y, et al. Diabetes is a risk factor for the progression and prognosis of COVID-19. Diabetes/Metabolism Research and Reviews. 2020: e3319.

10. Pan W, Zhang J, Wang M, et al. Clinical Features of COVID-19 in Patients With Essential Hypertension and the Impacts of Renin-angiotensin-aldosterone System Inhibitors on the Prognosis of COVID-19 Patients. Hypertension. 2020; 76: 732-741.

11. Kornum JB, Norgaard M, Dethlefsen C, et al. Obesity and risk of subsequent hospitalisation with pneumonia. Eur Respir J. 2010; 36: 1330-1336.

12. Kahlon S, Eurich DT, Padwal RS, et al. Obesity and outcomes in patients hospitalized with pneumonia. Clin Microbiol Infec. 2013; 19: 709-716.

13. Huttunen R, Syrjänen J. Obesity and the risk and outcome of infection. Int J Obesity. 2013; 37: 333-340.

14. China NHCO. the Diagnosis and Treatment of Pneumonia Infected by Novel Coronavirus(6th trial edition). February 19th, 2020.

15. Appropriate body-mass index for Asian populations and its implications for policy and intervention strategies. The Lancet. 2004; 363: 157-163.

16. Ministry of Health of the People's Republic of China. The guidelines for prevention and control of overweight and obesity in Chinese adults. (People's Medical Publishing House, 2006).

17. Wang $\mathrm{Y}$, Wang $\mathrm{L}, \mathrm{Qu} \mathrm{W}$. New national data show alarming increase in obesity and noncommunicable chronic diseases in China. Eur J Clin Nutr. 2017; 71: 149-150.

18. $\mathrm{Ng} \mathrm{M}$, Fleming $\mathrm{T}$, Robinson $\mathrm{M}$, et al. Global, regional, and national prevalence of overweight and obesity in children and adults during 1980-2013: a systematic analysis for the Global Burden of Disease Study 2013. The Lancet. 2014; 384: 766-781.

19. Dietz W, Santos Burgoa C. Obesity and its Implications for COVID-19 Mortality. Obesity. 2020; 28: 1005-1005.

20. Domínguez-Cherit G. Critically Ill Patients With 2009 Influenza A(H1N1) in Mexico. JAMA. 2009; 302: 1880. 
21. Kumar A. Critically Ill Patients With 2009 Influenza A(H1N1) Infection in Canada. JAMA. 2009; 302: 1872

22. Tsatsanis C, Margioris AN, Kontoyiannis DP. Association between H1N1 Infection Severity and Obesity - Adiponectin as a Potential Etiologic Factor. The Journal of Infectious Diseases. 2010; 202: 459-460.

23. Cai Q, Chen F, Wang T, et al. Obesity and COVID-19 Severity in a Designated Hospital in Shenzhen, China. Diabetes Care. 2020; 43: 1392-1398.

24. Price-Haywood EG, Burton J, Fort D, Seoane L. Hospitalization and Mortality among Black Patients and White Patients with Covid-19. New Engl J Med. 2020; 382: 2534-2543.

25. Palaiodimos L, Kokkinidis DG, Li W, et al. Severe obesity, increasing age and male sex are independently associated with worse in-hospital outcomes, and higher in-hospital mortality, in a cohort of patients with COVID-19 in the Bronx, New York. Metabolism. 2020; 108: 154262

26. Docherty AB, Harrison EM, Green CA, et al. Features of 20133 UK patients in hospital with covid-19 using the ISARIC WHO Clinical Characterisation Protocol: prospective observational cohort study. BMJ. 2020; 369: m1985.

27. Fan $\mathrm{H}$, Zhang X. Alarming Trends in Severe Obesity in Chinese Children from 1991 to 2015. Child Obes. 2020; 16: 244-249.

28. Zhang J, Wang M, Ding W, Wan J. The interaction of RAAS inhibitors with COVID-19: Current progress, perspective and future. Life Sci. 2020; 257: 118142.

29. Zhu L, She Z, Cheng X, et al. Association of Blood Glucose Control and Outcomes in Patients with COVID-19 and Pre-existing Type 2 Diabetes. Cell Metab. 2020; 31: 1068-1077.e3.

30. Yang $X, Y u Y, X u J$, et al. Clinical course and outcomes of critically ill patients with SARS-CoV-2 pneumonia in Wuhan, China: a single-centered, retrospective, observational study. The Lancet Respiratory Medicine. 2020; 8: $475-481$.

31. Wang Z, Ye D, Wang M, et al. Clinical Features of COVID-19 Patients with Different Outcomes in Wuhan: A Retrospective Observational Study. Biomed Res Int. 2020; 2020: 2138387.

32. de Heredia FP, Gómez-Martínez S, Marcos A. Obesity, inflammation and the immune system. P Nutr Soc. 2012; 71: 332-338.

33. Costa FF, Rosário WR, Ribeiro Farias AC, et al. Metabolic syndrome and COVID-19: An update on the associated comorbidities and proposed therapies. Diabetes \& Metabolic Syndrome: Clinical Research \& Reviews. 2020; 14: 809-814.

34. Wang M, Zhang J, Ye D, et al. Time-dependent changes in the clinical characteristics and prognosis of hospitalized COVID-19 patients in Wuhan, China: A retrospective study. Clin Chim Acta. 2020; 510: 220-227.

35. Qin C, Zhou L, Hu Z, et al. Dysregulation of Immune Response in Patients With Coronavirus 2019 (COVID-19) in Wuhan, China. Clin Infect Dis. 2020; 71: $762-768$.

36. Wang F, Nie J, Wang H, et al. Characteristics of Peripheral Lymphocyte Subset Alteration in COVID-19 Pneumonia. The Journal of Infectious Diseases. 2020; 221: $1762-1769$

37. DeFuria J, Belkina AC, Jagannathan-Bogdan $\mathrm{M}$, et al. B cells promote inflammation in obesity and type 2 diabetes through regulation of T-cell function and an inflammatory cytokine profile. Proceedings of the National Academy of Sciences. 2013; 110: 5133-5138.

38. Burton AR, Pallett LJ, McCoy LE, et al. Circulating and intrahepatic antiviral B cells are defective in hepatitis B. Journal of Clinical Investigation. 2018; 128: 4588-4603. 